\title{
Simulational study of anomalous tracer diffusion in hydrogels
}

\author{
Fatemeh Tabatabaei - Olaf Lenz - Christian Holm
}

October 22, 2018

\begin{abstract}
In this article, we analyze different factors that affect the diffusion behavior of small tracer particles (as they are used e.g. in fluorescence correlation spectroscopy (FCS)) in the polymer network of a hydrogel and perform simulations of various simplified models. We observe, that under certain circumstances the attraction of a tracer particle to the polymer network strands might cause subdiffusive behavior on intermediate time scales. In theory, this behavior could be employed to examine the network structure and swelling behavior of weakly crosslinked hydrogels with the help of FCS.
\end{abstract}

\section{Introduction}

Fluorescence Correlation Spectroscopy (FCS) is an experimental technique that allows to study the diffusion behavior of tracer particles in a surrounding medium in great detail.

The method is based on detecting the fluctuations of the fluorescent light intensity in a small and fixed volume element, usually formed by a laser focus of submicron size. There is a broad range of application for FCS, from studying the diffusive dynamics of simple colloids and polymers to biomolecules as well as hydrogels. [1,2, $3,4,5,6$

To describe the diffusion behavior of tracer particles, one typically uses the mean square displacement (MSD) $\left\langle|\Delta \mathbf{r}(t)|^{2}\right\rangle$, where $\mathbf{r}(t)$ is the average displacement of the

Fatemeh Tabatabaei · Olaf Lenz · Christian Holm

Institut für Computerphysik

Universität Stuttgart

Pfaffenwaldring 27

D-70569 Germany

E-mail: olenz@icp.uni-stuttgart.de particle after a time $t$. Often, the MSD of a particle behaves as

$\left\langle|\Delta \mathbf{r}(t)|^{2}\right\rangle \propto t^{\alpha}$

where $\alpha$ is the diffusion exponent. The case where the MSD scales linearly with time $(\alpha=1)$ is called normal diffusion, and is the most common case in physical processes, for example for particles that perform a simple random walk. A value of $\alpha \neq 1$ signifies anomalous diffusion, with the special cases of sub-diffusion $(\alpha<1)$ and superdiffusion $(\alpha>1)$. During the last decades, there has been considerable interest in anomalous diffusion, and such behavior has been found in many systems. [7, 8, 9, 10, 11, 12, 13

Fytas et al have employed the FCS technique to study the diffusion of tracer particles in a hydrogel [5]. They found evidence, that under certain circumstances, tracer particles in a hydrogel network exhibit anomalous diffusion behavior. 14

The goal of this work is to approach the problem from a theoretical point of view, to explain how anomalous diffusion behavior can occur in such a system, and the different processes that play a role in the diffusion. From the theoretical considerations, it is shown that FCS may be a valuable tool for examining the network structure and swelling behavior of real hydrogels.

Sprakel et al [15] have studied the diffusion of colloidal particles in polymer networks via photon correlation spectroscopy. They observe, that colloids that can bind to the polymer strands of the network show subdiffusive behavior. They explain this by the observation, that colloids that are bound to multiple polymer strands behave as though they are a part of the strand themselves, and thus show the Rouse-dynamics of a polymer segment. The authors develop a simple model that explains the observations. 
In our studies, the FCS tracer particles are smaller than the colloidal particles in the above work, and they are assumed to be significantly smaller than a network mesh cell. Furthermore, they do not bind to the polymer strands, although there might be attraction between the tracer and the network strands. To our best knowledge, there are no theoretical studies of this situation. In this work, we have focused on the behavior of tracer particles that are attracted to the polymer network and can slide along single strands. This gives rise to a number of interesting phenomena. The following thoughts are closely related to the biophysical problem of proteins sliding along DNA strands. [16, 17

\section{Processes affecting the diffusion behavior}

Several processes that can theoretically influence the diffusive behavior of the tracer particles in the hydrogel's polymer network can be identified (see Figure 1).

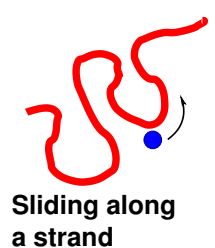

a strand
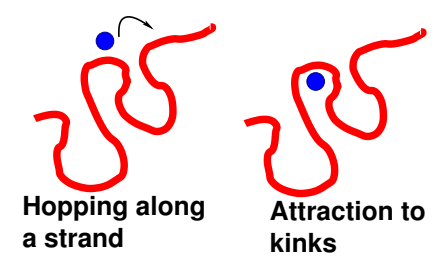
kinks

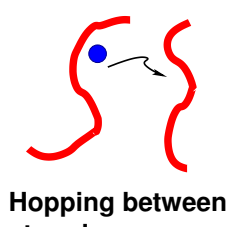
strands

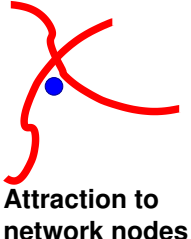

Fig. 1 Schematics of the different processes possibly involved in the diffusion of a small, non-specifically bound tracer particle in a hydrogel.

\section{Sliding}

From the point of view of this work, the most interesting process is the process of tracer particles sliding along a polymer strand. While tracer particles that are non-specifically bound to a polymer strand can not easily be detached from the strand by the random kicks of the solvent particles, they can still perform a onedimensional random walk along the strand. In that case, the particles can be considered as "diffusing" along the contour of the polymer. In this random walk, the mean contour length $c$ that the tracer has covered after the time $t$ behaves as

$\langle c\rangle \propto t^{\frac{1}{2}}$.
On the other hand, as it is well known since Flory's work [18, the contour of a relaxed polymer strand itself can be described as a random walk (RW) or a self-avoiding random walk (SAW) in three-dimensional space. It follows, that the MSD of a monomer $\left\langle|\Delta \mathbf{r}(t)|^{2}\right\rangle$ to another monomer with a distance $c$ along the contour behaves as

$\left\langle|\Delta \mathbf{r}(t)|^{2}\right\rangle \propto c$

in the case of the random walk, or as

$\left\langle|\Delta \mathbf{r}(t)|^{2}\right\rangle \propto c^{\frac{6}{5}}$

in the case of the self-avoiding random walk. Substituting equation 2 into equations 3 or 4 , this results in the $\operatorname{MSD}\left\langle|\Delta \mathbf{r}(t)|^{2}\right\rangle$ of the tracer particle behaving like

$\mathrm{RW}:\left\langle|\Delta \mathbf{r}(t)|^{2}\right\rangle \propto t^{\frac{1}{2}}$

or

$\mathrm{SAW}:\left\langle|\Delta \mathbf{r}(t)|^{2}\right\rangle \propto t^{\frac{3}{5}}$.

i.e. the diffusion exponents are $\alpha=\frac{1}{2}(\mathrm{RW})$ and $\alpha=\frac{3}{5}$ (SAW) respectively, which denotes strongly subdiffusive behaviour.

Equations 5 and 6 are only valid in the case of completely relaxed polymer coils. In the other extreme case, when the polymer is completely stretched and rod-like, the spatial distance of two monomers is identical to the contour distance of the monomers. Tracer particles sliding along such a rod exhibit normal diffusive behavior $(\alpha=1)$. Consequently, when stretching a polymer and thus crossing over from relaxed coil states to stretched rod-like polymer states, a transition from subdiffusive to normal diffusive behavior must take place. Note, that in both cases the diffusion exponent applies to all length and time scales.

When the polymer strand is neither fully stretched nor fully relaxed, the polymer structure is becoming more complex. Stretching the polymer chain does not affect the polymer structure on all length scales equally. The structure of the stretched polymer on short length scales will be unaffected by the stretching and retain the random-walk or self-avoiding random walk behavior, while on longer length scales, the polymer is stretched and becomes rod-like. A simple description of this behavior is given by Pincus' blob picture. In that picture, a polymer chain under tension can be seen as a chain of blobs, each of which contains a distinct segment of the polymer. While the chain of blobs behaves rod-like, each of the blobs itself consists of a polymer segment that can still be described well by a RW or SAW. The size of the blobs is determined by the stretching; the larger the amount of stretching, the smaller the blobs. 19, 20 
When applying this picture to the behavior of the tracer particles that slide along the polymer contour, the MSD of the tracer particles can be expected to show a more complex behavior at different time scales, which would be characterized by varying diffusion exponents at different time scales. At small time scales, the tracer particles probe the random-walk-like internal structure of the Pincus blobs, and the subdiffusive behaviour described above should be observable, with small exponents down to $\alpha=\frac{1}{2}$ (RW) and $\alpha=\frac{3}{5}$ (SAW), respectively. At longer time scales, the tracer particles probe the stretched structure of the sequence of Pincus blobs, which leads to normal diffusive behavior and an exponent of $\alpha=1$.

However, formally, the diffusion exponent $\alpha$ is only defined asymptotically for large timescales, therefore it is not very useful in our case. To be able to distinguish more details of the diffusion behavior at different time scales, we instead examine the time-dependent exponent $\alpha(t)$

$\alpha(t)=\frac{\mathrm{d} \log \left\langle|\Delta \mathbf{r}(t)|^{2}\right\rangle}{\mathrm{d} \log t}$

In the case of hydrogels, the degree of stretching of the polymer strands is directly influenced by the swelling ratio of the hydrogel. While in a dry or only slightly swollen hydrogel, the strands can be expected to be mostly relaxed, the strands must be stretched for strongly swollen hydrogels. This means, that the diffusion behavior of non-specifically bound tracer particles should exhibit the characteristics described above and should change accordingly when the gel is swollen.

If this effect occurs in the parameter range of real hydrogels and is not dominated by other processes that influence the diffusion behavior of the tracer particles, it could provide a nice way to explore the structure of the polymer network and the swelling of hydrogels via FCS. One should note, however, that this method only works if the average length of the polymer segments (which corresponds to the average network mesh cell size) and the average time required to slide along these segments are larger than the minimal time and length resolution of the FCS technique. For most real hydrogels, this is not the case. Only in weakly crosslinked hydrogels, it can be expected that the mesh size comes into regions where this effect might be observable.

\section{Hopping}

The above discussion only holds for an ideal model where the tracer particles are sliding along the contour of the polymer. In reality, however, a number of other processes play a role in the diffusion behavior of tracer particles.

When the tracer particles are not very strongly attracted or bound to the polymer strand, they can be ripped off by thermal motion and diffuse freely in the solvent until they are adsorbed again by the same or another polymer strand. This process of escape and readsorption is referred to as hopping throughout this work. There are actually two hopping processes; the tracer particle can be reabsorbed by the same network strand as it was ripped off, which is referred to as hopping along a strand, or it is absorbed by another polymer strand, which is called hopping between strands.

When hopping occurs, the tracer particle diffuses freely in the solvent. Compared to the sliding process depicted above, it can be expected that free diffusion has a significantly larger diffusion constant than the sliding process and a diffusion exponent of $\alpha=1$, as it is a normal diffusive process. Since the diffusion of the hopping particles is much faster than of the sliding particles, there is the danger that it dominates the overall diffusive behavior and effectively hides the anomalous behavior of the sliding process. The relative importance of hopping towards sliding is controlled by the following parameters:

- The strength of the attractive interaction governs the hopping rate: the stronger the attraction, the lower the probability for the tracer to escape from the polymer strand, and the higher the probability to get readsorped. This parameter affects both hopping processes.

- The density of the polymer strands directly controls the mean free path that the tracer can cover before it gets absorbed by another polymer strand, i.e. it influences the effect of hopping between strands.

- The spatial correlations between different parts of the polymer strands are unequal in the coiled and stretched states. In the coiled state, a tracer particle is more likely to be readsorped onto another part of the same polymer strand, as in the stretched state. This means that the process of hopping along a strand is suppressed by stretching the strand, while hopping between strands should be mostly unaffected.

\section{Sticking}

It is likely that network nodes or kinks in a polymer strand, where tracer particles can synchronously interact with multiple polymer segments further affect the diffusive behavior. The strong attraction of the tracer particles to the polymer strand at these locations might 
immobilize the tracer particles, which reduces the overall diffusion rate. 13

\section{Models}

Starting from the assumption that tracer particles can slide along the polymer contour and therefore show subdiffusive behavior, the goal of this work was to study different influence factors on this behavior with the help of simulations, to quantify them and their relation to each other, and to distinguish their different origins.

To this end, we have developed a series of coarsegrained, simplified diffusion models of the system where we successively add details and allow more and more of the processes depicted above to happen.

All of the diffusion models used fixed polymer conformations, i.e. the polymer did not move throughout the diffusion simulation. This simplifies the models to a great extent, as it takes out the influencing factors of the various parameters of the polymer simulation, at the cost of not describing correctly the influence of the polymer dynamics on the tracer particles. We believe that the dynamics of the polymer will not influence the diffusive behavior of the tracer particles to a great extent, although it might shift some of the results obtained in this work.

Furthermore, in the models used in this work, we did not consider the case of a polymer network with connections between different polymer strands, but we always used a single polymer strand of infinite length. Consequently, we did not observe the effect of network nodes on the diffusion behavior. We plan to consider both the effect of the dynamics of the polymer as well as the effect of a polymer network with network nodes in our future work.

\section{Polymer model}

All of the diffusion models used conformations of an coarse-grained bead-spring polymer with different degrees of stretching. The conformations have been generated via a standard Langevin dynamics simulation of a three-dimensional model polymer system with periodic boundary conditions.

The polymer consisted of $N=200$ beads (or "monomers each with mass $m=1$, that were bound to each other using a harmonic spring potential to form a chain.

$V_{\text {harmonic }}(r)=\frac{1}{2} K\left(r-r_{0}\right)^{2}$

The parameters were chosen to be $r_{0}=0.5$ and $K=$ 10.0 .

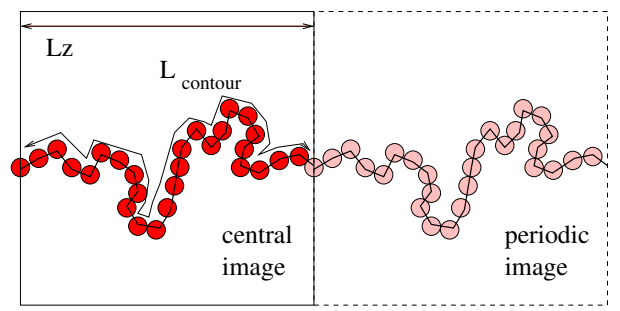

Fig. 2 Sketch of the system with a stretched periodically repeated polymer. The degree of stretching is defined by the number of monomers $N$ and the system size along the axis of stretching $L_{z}$.

To generate a polymer of seemingly infinite length with various degrees of stretching, the polymer was periodically repeated along the $z$-axis of the system, i.e. the last monomer of the chain was bound to the first monomer of the chain over the periodic boundary (see figure 2). Note that this does not mean that any of the beads was fixed in any spatial dimension throughout the simulations.

In this model, the degree of stretching depends on the number of monomers $N$ and the size of the system along the $z$-axis $L_{z}$. Given a value of $L_{z}$, the system lengths $L_{x}=L_{y}$ could be used to control the monomer density $\rho$ of the system.

The unusual setup of the periodically repeated polymer also had some effects on the Pincus blob picture mentioned above. On the one hand, the system size $L_{z}$ limited the maximal size of a Pincus blob. As the various periodic images were always aligned chain-like, the polymer always displayed a rod-like structure for length scales larger than $L_{z}$. Accordingly, the time-dependent diffusion exponent $\alpha(t)$ always approached 1 when the tracer particles diffused further than this length scale. It should be noted that this was an artifact of the simulation setup, which limited the maximal Pincus blob size. In real systems with fully relaxed chains, it should in theory be possible to see the subdiffusive behavior on larger length and time scales. On the other hand, when $L_{z}$ was chosen smaller than the expected size of a completely relaxed polymer coil (given by Flory's argument), this corresponds to an unphysical compression of the polymer in this direction. Therefore, such states were avoided throughout this work.

Two variants of the polymer model were simulated. In the first variant, no excluded volume interaction between monomers was used, therefore the conformations in the coiled states correspond to a random walk (RW). The RW conformations were used only in conjunction with the strongly bound tracer model described below to verify the theoretical considerations above. In the second variant, a repulsive Weeks-Chandler-Anderson (WCA) interaction between the monomers was used to 
model the excluded volume interaction [21,22, which is identical to the repulsive core of the Lennard-Jones interaction cut off at its minimum (equation 9, $\varepsilon=1.0$, $\sigma=1.0$ ). In this case, the conformations corresponded to a self-avoiding random walk (SAW) [23].

$V_{\mathrm{WCA}}(r)= \begin{cases}4 \varepsilon\left(\left(\frac{\sigma}{r}\right)^{12}-\left(\frac{\sigma}{r}\right)^{6}\right) & , \text { if } r<(2 \varepsilon)^{\frac{1}{6}} \\ 0 & , \text { otherwise }\end{cases}$

The system was simulated with help of the simulation package ESPRESSo [24, using the Velocity Verlet algorithm with a time step of $\tau=0.005$ and a Langevin thermostat with temperature $T=1.0$ and a friction parameter $\gamma=0.5$. Various simulation runs with up to $50 \times 10^{6} \tau$ in total and $500,000 \tau$ between single polymer conformations were performed, to obtain 100 equilibrated, statistically independent polymer conformations for each different value of the number of monomers $N$, degree of stretching (i.e. $L_{z}$ ) and polymer density $\rho$. Note that $\rho$ was always chosen small enough so that the different periodic images of the polymer strand in $x$ or $y$ direction did not interact with each other. For the given parameters, the expected end-toend distance (and hence the value of $L_{z}$ ) of the RW polymer is about 12, while for the SAW polymer it is about 20 (given the measured average bond length of about 0.7 ). This means that the polymer conformations at $L_{z}=15$ (RW) resp. $L_{z}=30$ (SAW) correspond to mostly relaxed polymer chains, while polymer conformations at $L_{z}=100$ (RW and SAW) are strongly stretched.

\section{Strongly bound tracer model}

In the simplest diffusion model, only the process of the tracer particle sliding along the polymer contour was considered. To this end, the frozen polymer conformations were used as a basis for a one-dimensional random walk along the polymer contour, where the tracer particles were thought to be strictly bound to the polymer and could not escape.

To do that, a random monomer from each conformation was used as a first binding location of the virtual, strongly bound tracer particle. When advancing a "timestep", either the same, the next or the previous monomer along the chain was randomly chosen as the next binding location of the virtual particle, to model the diffusion of the tracer along the polymer. After $t$ timesteps, the MSD of the binding location was measured to determine the spatial diffusion behavior of the virtual tracer.

The model was simulated for the various conformations obtained in the polymer model, and the dependence of the diffusive behavior of the virtual tracer on the degree of stretching $\left(\right.$ i.e. $\left.L_{z}\right)$ and the chain length $N$ was studied. As the virtual tracer particle could not leave the single infinite strand, and the different periodic images of the polymer strand did not interact with each other, the monomer density $\rho$ does not play a role in this model.

The results were used to verify the theoretical considerations from above. Therefore, the model was simulated both with the RW polymer conformations as well as the SAW polymer conformations.

Freely diffusing tracer particles with nearest-neighbor interaction

On the next level of model refinement, the influence of the hopping process onto the diffusive behavior of the tracer particle was studied. The model was refined, and a simple model of a single tracer particle was introduced. The tracer particle was represented by a bead of mass $m=1$ that interacted with the (fixed) polymer beads via a Lennard-Jones interaction (equation $10, \sigma=1.0, r_{\text {cut }}=2.0, \varepsilon$ varied). As the polymer beads were fixed in space, this can also be interpreted as the diffusion of the tracer particle in a system of fixed obstacles.

$V_{\mathrm{LJ}}(r)= \begin{cases}4 \varepsilon\left(\left(\frac{\sigma}{r}\right)^{12}-\left(\frac{\sigma}{r}\right)^{6}\right) & , \text { if } r<r_{\text {cut }} \\ 0 & \text {, otherwise }\end{cases}$

On this refinement level, we still wanted to avoid the effect of the tracer particle getting stuck at kinks of the polymer strand, where the bead would feel a significantly deeper potential well than close to a stretched piece of the polymer. To achieve that, we have modified the interaction such, that the tracer bead only interacted with the single monomer on the polymer strand that was closest to the tracer particle. We call this the nearest-neighbor interaction. This simple trick prevented the tracer particle from getting stuck at kinks, while it still felt the attraction of the strand.

The most important parameter introduced by this model was the parameter $\varepsilon$ of the interaction, which determined the strength of the attraction between the tracer particle and the polymer and consequently the rate of the hopping process. Compared to a system with an all-neighbor LJ interaction, the value of $\varepsilon$ had to be chosen much larger, as in this model the tracer particle always only feels the attraction of a single monomer as opposed to several monomers at once when being close to the strand in a normal Lennard-Jones all-neighbor interaction. Another parameter that influenced the behavior of this model was the monomer density $\rho$, which mostly controlled the rate of the hopping between polymer strands. 
Langevin MD simulations (time step $\tau=0.01$, temperature $T=1.0$, friction $\gamma=0.5$ ) of the tracer were performed. The MSD and the time-dependent exponent $\alpha(t)$ of the tracer diffusion was measured. The model was studied for various SAW polymer conformations at different degrees of stretching $L_{z}$ and different chain lengths $N$, as well as at different monomer densities $\rho$. The RW polymer conformations were not considered in this model.

It is important to note that there is no relation between the time scale in this model and the time scale in the strongly bound tracer. While the time scale in the strongly bound tracer model was a pseudo time scale in units of the time that the tracer particle needs to diffuse to the next monomer in the polymer chain, the time unit in this model is determined by the LJ parameters $\varepsilon$ and $\sigma$.

Freely diffusing tracer particles

The last model considered in this work was mostly identical to the previous model with nearest-neighbor interaction, but used a conventional all-neighbor LennardJones interaction (Equation 10, so that the tracer particle interacted with all polymer beads within cutoff range. Thus, the effect of the tracer particle getting stuck to kinks was included in this model.

The simulation parameters are similar to the previous model. The only difference is the depth of the Lennard-Jones potential $\varepsilon$, which had to be chosen significantly lower in this model to exhibit a comparable effect.

\section{Results}

\section{Strongly bound tracer model}

The upper subplot of figure 3 shows the MSD of the tracer particles in the strongly bound tracer model, for both RW and the SAW polymers as well as for two different representative values of stretching of the chain, while the lower subplot shows the time-dependent exponent $\alpha(t)$ in the same cases. While for the relaxed polymer conformations $L_{z}$ was close to the expected end-to-end distance ( $L_{z}=15$ in the RW and $L_{z}=30$ in the SAW case), for the stretched conformations it was rather large with $L_{z}=100$.

As expected, the plots show a constant diffusion exponent $\alpha(t)=1$ for a linear chain of monomers, while for the polymer chains it varies between $\frac{1}{2}<\alpha(t)<1$ for the polymer in the RW case and $\frac{3}{5}<\alpha(t)<1$ in the SAW case. Interestingly, all of the curves for RW and

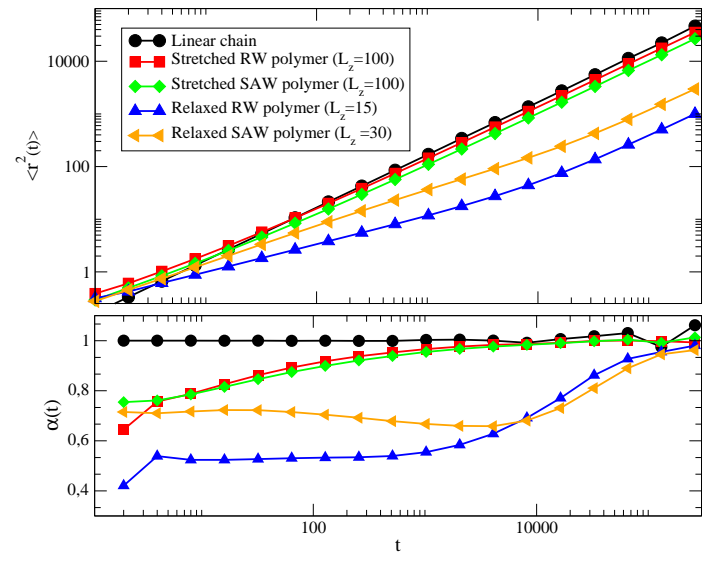

Fig. 3 Top: MSD of the strongly bound tracer model for both polymer model variants (RW and SAW) at two different degrees of stretching. Bottom: Time-dependent exponent of the curves in the top plot.

SAW polymers alike show values of the time-dependent exponent $\alpha(t)$ at small time scales that are significantly less than 1 , and approach a value of 1 at longer time scales. This behavior is consistent with the picture of the strongly bound tracer particle in a polymer that consists out of Pincus blobs - at short time scales, the tracer probes the coiled structure of single Pincus blobs and exhibits subdiffusive behavior, while at longer time scales, the tracer particles feel the linear alignment of multiple Pincus blobs, which results in normal diffusive behavior.

In the case of the stretched polymers (both RW and SAW), the time-dependent exponent quickly approaches a value of 1 within a few orders of magnitude of the time scale, while in the case of the relaxed polymers, it stays at values of $\alpha(t) \approx 0.5(\mathrm{RW})$ or $\alpha(t) \approx \frac{3}{5}$ (SAW) over many orders of magnitude. This is also well described by the Pincus blob picture, which predicts that even while the overall structure of the polymer is unaffected by stretching the polymer, the single blobs are largest for relaxed polymer coils, while for higher degrees of stretching the blobs become smaller. Note, that even in the maximally relaxed polymer coils in our model the time-dependent exponent $\alpha(t)$ has to approach a value of 1 for long time scales $t$, as the Pincus blob is limited by the system size $L_{z}$ as described above.

Freely diffusing tracer particles with nearest-neighbor interaction

In order to investigate whether it is at all possible to observe an effect of the polymer structure on the diffusive behavior of the tracer particles in the tracer model 


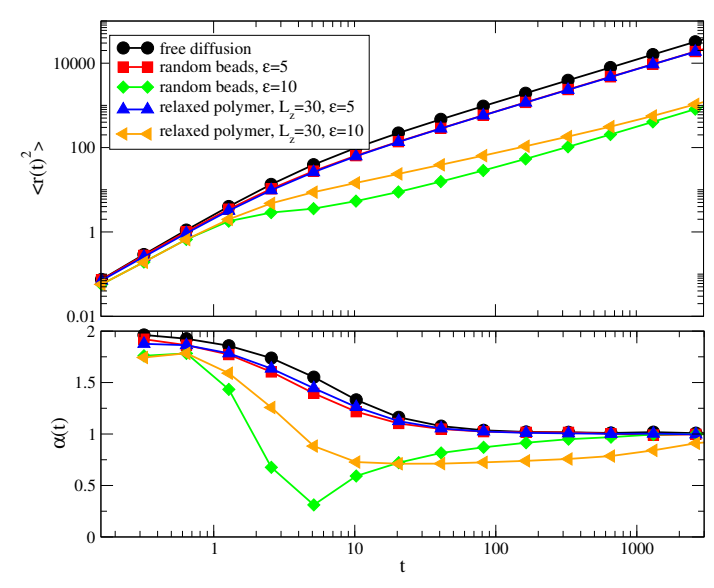

Fig. 4 MSD and time-dependent exponent of the freely diffusing tracer model with nearest-neighbor interaction in a system without any obstacles ("free diffusion"), in a system of random beads at two different interaction strengths, and in a system with a relaxed polymer chain at two different interaction strength. The density of the random beads was chosen to match the polymer results as described in the text.

with nearest-neighbor interaction, we have performed a few simulations of systems that contained randomly distributed beads as obstacles. If the polymer structure has an effect, the diffusion behavior should show distinct differences in such a system when compared to a system of random beads. To be able to do such a comparison, it is important to note that the beads in the chain have a significant overlap, while random beads do not. Therefore, the volume fraction of the system where the tracer particle interacts with an obstacle bead is significantly larger in the case of the random beads than in the case of the polymer chain at similar obstacle bead density. To be able to see whether the actual structure of the polymer has a distinct influence on the diffusive behavior, it is therefore necessary to match the volume affected by the obstacle beads rather than the density.

The MSD and time-dependent exponent of tracer particles of different systems of random beads and a relaxed polymer chain are plotted in Figure 4. These plots differ significantly from the corresponding plots in the strongly bound tracer model. The most obvious difference is that at very short time scales of $t<1$, the MSD seems to exhibit strong superdiffusion with an exponent of up to 2 , that was not visible in the strongly bound tracer model. This behavior is an artifact of the tracer MD simulation; even in the case of free diffusion, on these short time scales, the tracer particles propagated freely and exhibited ballistic motion with a diffusion exponent of 2, before the Langevin thermostat destroyed the velocity autocorrelation of the tracer particles and the normal diffusion sets in. This effect is of no inter- est to this work, therefore none of the figures following figure 4 show this regime.

At relatively low interaction strength $\varepsilon=5$, only a very minor effect of the random obstacle beads on the diffusive behavior is observed, and the MSD plot shows the same two clearly distinct regimes - a shorttime ballistic regime, and a longer-time normal diffusive regime. The differences can be attributed to the beads acting as simple obstacles that hinder the diffusion of the tracer and thus reduce the diffusion constant. This also holds in the case of the relaxed polymer chain at low interaction strength, where the MSD is virtually identical to the random bead system. No effect of the polymer structure can be recognized.

When the interaction strength is increased, the effect of the random beads hindering the diffusion becomes more pronounced, up to a level where the normal diffusion is so slow that between the ballistic and normal-diffusive regime a short intermediate region appears, where the time-dependent exponent drops below 1. This region should not be interpreted as a real subdiffusive regime, instead it is more of an artifact caused by the combination of two normal diffusion processes with very different length and energy scales: the diffusion within the potential of a single bead is very fast, but the tracer is strongly bound to the bead ("rattling in a cage"). When the tracer particle escapes, it can diffuse fast and freely until it is caught by another attractive bead ("hopping between cages"). Looking at the overall diffusion, this results in the observed behavior with the pseudo-subdiffusive intermediate region.

In the case of the relaxed polymer chain at high enough interaction strength, the effect is different. As in the case of the random beads, an intermediate region occurs between the ballistic and normal-diffusive regimes, where the time-dependent exponent is below 1 . In contrast to the random bead system, however, $\alpha(t)$ is mostly constant and below 1 for several orders of magnitude, so that we can speak of a real subdiffusive regime.

When examining the MSD plot in more detail, it can be observed that in the case of the relaxed polymer chain, this subdiffusive regime spans time scales of up to at least 1000 time units and the corresponding length scale of up to a few tens of bead diameters, indicating that the tracer bead really slided along the polymer contour for a few tens of monomers.

Plotting the MSD and time-dependent exponent is very useful to look at the overall diffusion behavior. A more detailed way to visualize what is going on in a system is to plot the displacement probability distribution $P(r)$ at different times. As we are mainly interested in the diffusion of the tracer particles along the 


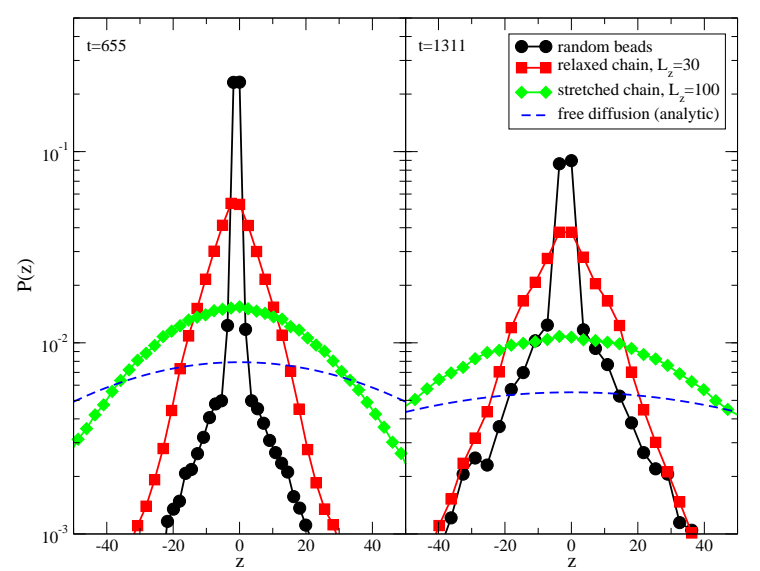

Fig. 5 Probability distribution of the tracer displacement in $z$ direction at two different times. Other parameters: interaction strength $\varepsilon=10$, density $\rho=0.0084$. The free diffusion case was computed analytically using Equation 11

$z$-axis of the system, Figure 5 depicts the displacement probability distribution $P(z)$ for different systems at different time scales in the intermediate regime where the tracer particles exhibit subdiffusion in some of the systems. In the case of free diffusion, we can easily compute the displacement distribution function analytically as in Equation 11, 25, 26] When plotting the measured displacement probability in the free diffusion case, it is indistinguishable from the analytically derived function.

$P(z, t)=\frac{1}{\sqrt{4 \pi D t}} \exp \left(\frac{-z^{2}}{4 D t}\right)$.

In the plot, we can discern the qualitative differences between the various systems. While the free diffusion case shows Gaussian behavior on all time scales, in the other systems we can observe a more complex behavior. The distributions in the random bead system are characterized by a sharp peak at $z=0$ with a very small width and lower, much broader shoulders. This behavior is consistent with a superposition of a sharp peak and a broader Gaussian, where the peak originates from the tracer particles being caught in the potential of a random bead, while the smaller but significantly broader Gaussian comes from the free diffusion of particles that have escaped the bead potentials. The distributions measured in the polymer systems look distinctively different from both of these extremes. None of the curves looks like a clear superposition of two Gaussians, but none of them really looks like a Gaussian either.

To determine the effect of the degree of stretching of the polymer on the diffusion behavior, we have performed a number of simulations for varying degrees of stretching at constant density $\rho=0.0084$ and constant interaction strength $\varepsilon=10$ (Figure 6). We observe, that

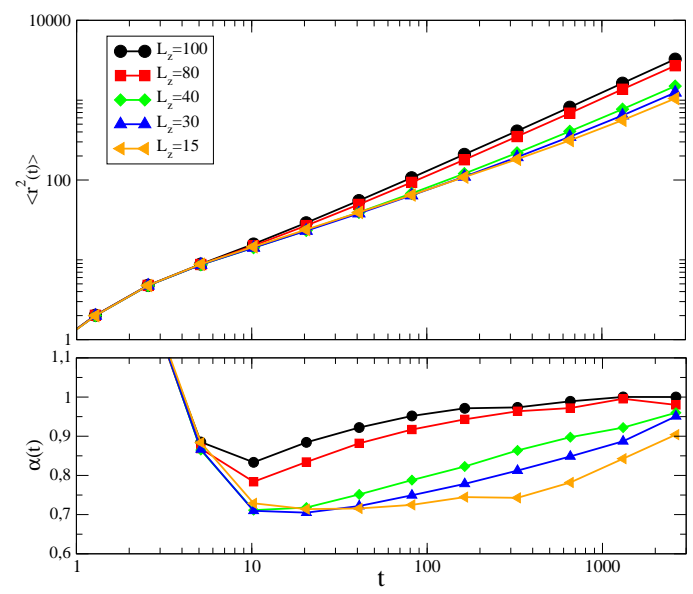

Fig. 6 MSD and time-dependent exponent of the freely diffusing model tracer with nearest-neighbor interaction for different degrees of stretching of the polymer at constant density $\rho=0.0084$ and interaction strength $\varepsilon=10$.

the size of the intermediate regime seems to depend on the degree of stretching of the attractive polymer chain in the system; for mostly stretched chains, the normaldiffusive regime is restored after a few orders of magnitude of the time scale, while for relaxed chains, the MSD shows subdiffusive behavior for many decades. In the most relaxed chains, the normal-diffusive regime is restored at about the length scale of $L_{z}$, which corresponds to the fact that the Pincus blob size is limited by $L_{z}$. Therefore, this behavior is again well consistent with the Pincus blob picture.

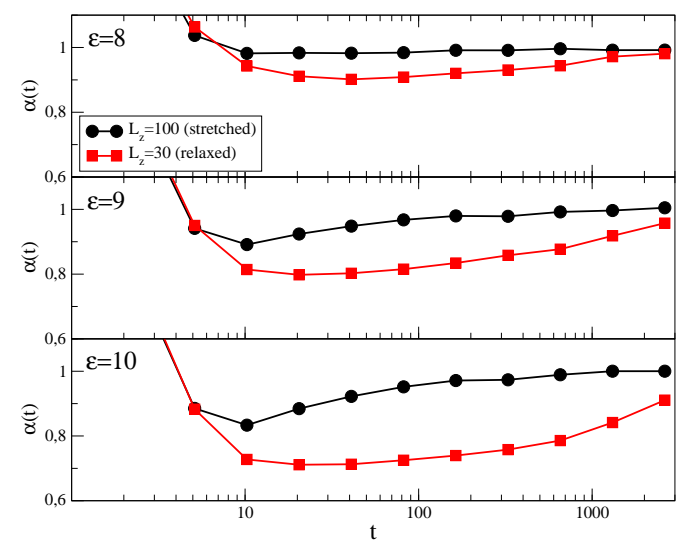

Fig. 7 Time-dependent exponent for two different degrees of stretching $\left(L_{z}=30\right.$ and $\left.L_{z}=100\right)$ at different values of the interaction parameter $\varepsilon$ and constant monomer density $\rho=0.0084$.

Further simulations have been performed for varying values of the interactions strength $\varepsilon$ (Figure 7 ) and the monomer density $\rho$ (Figure 8), to be able to quan- 


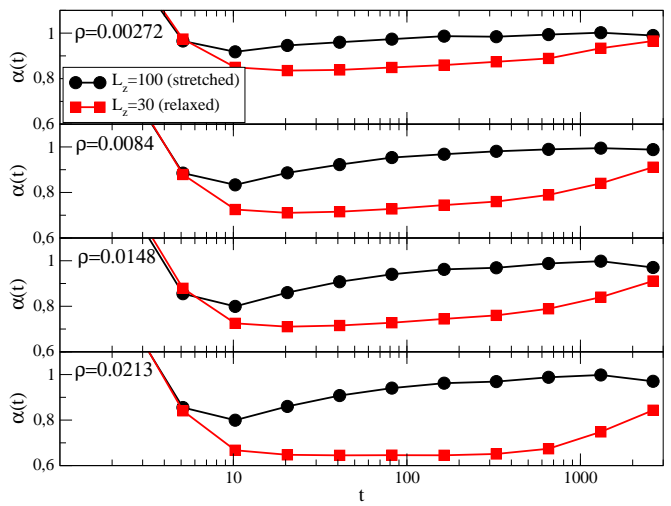

Fig. 8 Time-dependent exponent for two different degrees of stretching $\left(L_{z}=30\right.$ and $\left.L_{z}=100\right)$ at different values of the monomer density $\rho$ and constant interaction parameter $\varepsilon=10$.

tify the influence of these parameters on the diffusion behavior. From the plots, it can be concluded that lowering the interaction strength $\varepsilon$ suppresses the effect of the polymer chain, as does lowering the density $\rho$. As discussed in the introduction, this can be traced to the influence of the hopping process which dominates the diffusion along the polymer. Although the qualitative effect of varying the interaction strength $\varepsilon$ (Figure 7) is similar to varying the density $\rho$ (Figure 8), quantitatively the interaction strength has a significantly higher impact on the subdiffusive regime, where varying $\varepsilon$ by $20 \%$ has a similar effect to varying $\rho$ by factor 5 . We interpret this along the lines of what was discussed in the introduction - while varying $\rho$ only has an effect on the less important process of hopping between polymer strands, the interaction strength has an effect on all hopping processes, both on the more important process of hopping along the same strand as well as on hopping between strands.

Freely diffusing tracer particles

Figure 9 depicts the MSD and time-dependent exponent of freely diffusing tracer particles at different degrees of stretching $L_{z}$ at a density of $\rho=0.0084$. Comparing these plots to the corresponding plots in the model with nearest-neighbor interaction reveals a number of profound differences. The ballistic behavior seen at short times disappears after even shorter times than in the nearest-neighbor interaction model. We think that the effect is caused by the introduction of kinks into the model. When employing the usual all-neighbor Lennard-Jones interaction, the tracer feels the deep potential wells along the polymer chain where the chain

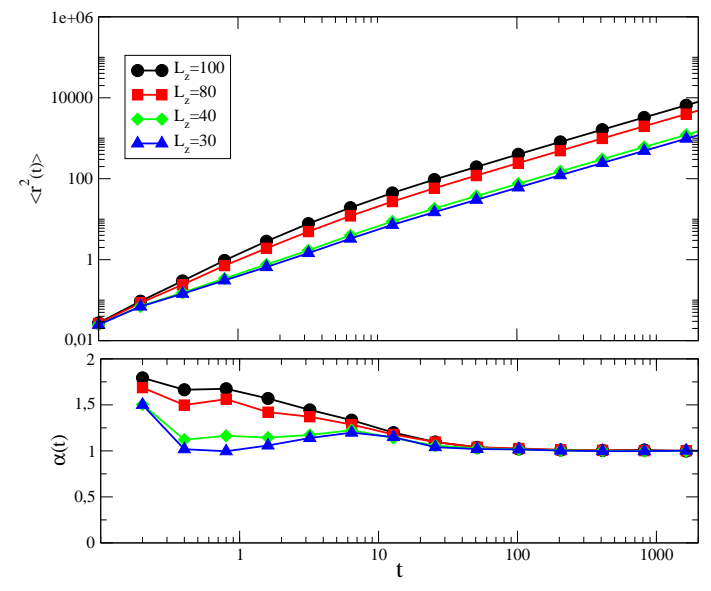

Fig. 9 MSD and time-dependent exponent of the freely diffusion model tracer for different degrees of stretching of the polymer at interaction strength $\varepsilon=2$ and density $\rho=0.0084$.

has a kink, and can easily be trapped in them for long times (see Figure 10). Since the potential well caused by the kink is very narrow, it affects the ballistic behavior of the tracer particle on very short time scales, even before the velocity autocorrelation is destroyed by the Langevin thermostat.

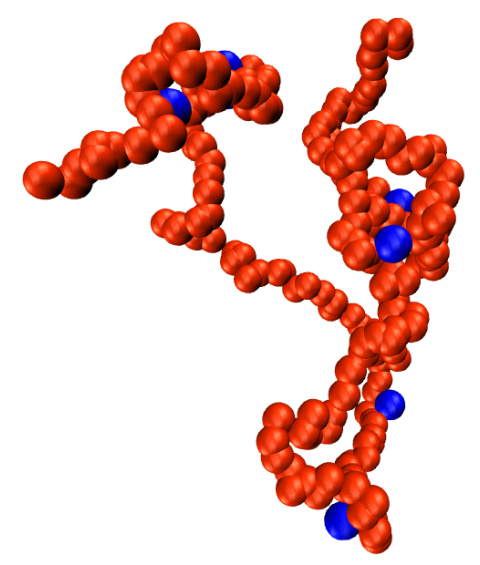

Fig. 10 Simulation snapshot of the tracer particles getting stuck in the kinks of a coiled polymer.

Curiously, the plots of the time-dependent exponent at low density still allows to distinguish between the different degrees of stretching. The reason for this is, that the number of kinks is significantly larger for 
a coiled polymer chain than for a stretched polymer chain. Therefore, for very coiled polymer chains, $\alpha(t)$ drops much faster than for a stretched chain. This is, however, not the effect of the tracer particles sliding along the polymer contour and shall not be further considered in this work.

The subdiffusive regime that was observed in the nearest-neighbor model at similar densities has completely disappeared. From the ballistic regime at very short times, the time-dependent exponent directly goes over into a normal diffusive regime. We believe that in these systems the anomalous diffusion caused by the tracer particles sliding along the chain is on the one hand completely dominated by the process of particles being immobilized by the kinks, and on the other hand by the process of hopping, and therefore it is not visible in these graphs.

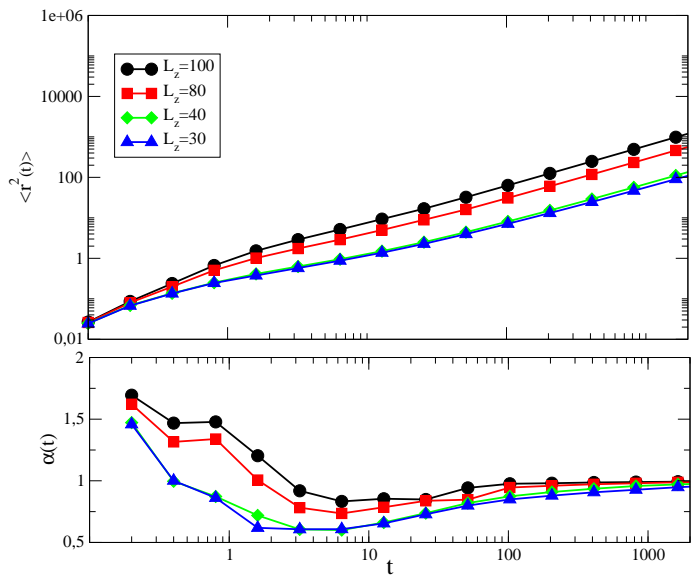

Fig. 11 MSD and time-dependent exponent of the freely diffusion model tracer for different degrees of stretching of the polymer at interaction strength $\varepsilon=2$ and density $\rho=0.1$.

To increase the importance of tracer particles sliding along the chain over the process of hopping and sticking, we have increased the density to significantly higher values of $\rho=0.1$, which should decrease the importance of hopping. The MSD and time-dependent exponent are plotted in Figure 11 . In these plots, the timedependent exponent $\alpha(t)$ drops below 1 in an intermediate region, which might indicate subdiffusive behavior. However, the subdiffusive behavior already sets in at very short times, where the tracer particles have not yet diffused even a single bead diameter. Therefore at least this part of the subdiffusive region can not originate in the tracer particles probing the chain contour. Instead, it can be assumed that it is a result of a "hoppingbetween-cages"-process similar to the effect observed in the random bead system in the nearest-neighbor in- teraction model, only that in this case the cages are represented by the kinks rather than by single beads.

Unfortunately, the plots do not allow to celarly decide whether subdiffusive behavior caused by the tracers sliding along the polymer contour is visible within the system. Since the densities used for producing the data of these figures are very high, an experimental verification of these features seem to be very improbable.

\section{Conclusions}

In this work, we have investigated the effect of polymer strands (as they might be found in a hydrogel) on the diffusion behavior of small tracer particles (as they are used in FCS) that are attracted to the polymer strands. From theoretical considerations, we concluded that when such tracer particles slide along the contour of a polymer strand, they will exhibit subdiffusive behavior due to the fractal nature of the polymer.

To investigate this situation, we have devised a series of simplified models with increasing complexity. A simple numerical simulation of a model with tracer particles that were strongly bound to frozen conformations of a polymer strand proved the soundness of the theoretical assumptions. In plots of the mean square displacement (MSD) and the time-dependent exponent, it was observed, that when the polymer is stretched (i.e. when the hydrogel is swollen), this affects the structure of the polymer contour and the subdiffusive behavior of the sliding tracer particles. This is best rationalized in terms of Pincus blobs. When the polymer strands are mostly relaxed, the blobs are relatively large, and the subdiffusive behavior of the tracer particles should be observable over many decades of the time scale, while when the polymer strands are stretched, the Pincus blobs become small and the subdiffusive behavior can only be observed on very short times.

In reality, tracer particles cannot be expected to be so strongly bound to the polymer. Instead, thermal kicks induced by the polymer motion most probably will aid their desorption and particles can be expected to diffuse freely between the polymer strands, which is referred to as "hopping". The influence of this process was investigated by the means of a simple molecular dynamics simulation model, where the tracer particles interacted with the frozen polymer conformations via a nearest-neighbor Lennard-Jones interaction. We observed, that the hopping process easily dominates the overall diffusion behavior, as it has a much higher diffusion rate than the process of sliding along the polymer. To be able to see the subdiffusion caused by the tracer particles sliding along the polymer, the most important 
parameter was determined to be the strength of the attractive interaction between the tracer and the polymer chain, while the density of the polymer strands was of minor importance.

In the last model, where the interaction between tracer particles and the frozen polymer was modeled by a standard all-neighbor Lennard-Jones interaction, we noticed that kinks within the polymer chain have a profound impact on the diffusion behavior. The reason is the increased attraction of the tracer particles to the kinks, which immobilizes the tracer particles and dominates the diffusion behavior. At low densities, the subdiffusive behavior caused by the tracer particles sliding along the chain is completely hidden. Although at high density, an apparently intermediate subdiffusive region appears, we noticed that it occurs at time and length scales that are still within the direct vicinity of the kinks. Therefore we think it is caused by hopping between the kinks.

We believe that the large effect of these kinks on the diffusive behavior is not as dominating in real hydrogel systems, but instead can be seen as an artifact of our model which originates in the fact that we use fixed polymer conformations in our model. In the real world, where the polymer is moving, potential wells caused by kinks should be too short-lived to really catch tracer particles.

We should remark that the parameters of the simulational model systems are applicable only to weakly crosslinked hydrogels, since normal hydrogels have mesh sizes of about tens of $\mathrm{nm}$. This means that the experimentally accessible length and time scales are larger than the average network mesh size and hence the length of the pieces of polymer strands that the tracer can slide along. Experimentally, one should be able to see the simulated anomalous effects only in weakly crosslinked hydrogels that have large enough network mesh cells.

As final conclusion we believe that further studies are required to determine whether it is possible to explore the polymer structure in a hydrogel by the means of tracer diffusion studies.

Acknowledgements We acknowledge the financial support provided by the Deutsche Forschungsgemeinschaft (DFG) as part of the SPP 1259 "Intelligente Hydrogele".

The authors want to thank Georg Fytas, Alexander Grosberg, Owen Hickey, Felix Höfling, Peter Košovan, Ralf Metzler, Ricardo Raccis, and Roland Winkler for helpful discussions.

\section{References}

1. R.G. Winkler, J. Chem. Phys. 127, 054904 (2007) (document)
2. D. Lumma, S. Keller, T.Vilgis, J.O. Rädler, Phys. Rev. Lett. 90, 218301 (2003) (document)

3. C.K. Nandi, P.P. Parui, B. Brutschy, T.L. Schmidt, A. Heckel, Anal. Bioanal. Chem 390, 1595 (2008) (document)

4. K. Bacia, D. Scherfeld, N. Kahya, P. Schwille, Biophys. J. 87, 1034 (2004) (document)

5. T. Cherdhirankorn, A. Best, K. Koynov, , K. Peneva, K. Müllen, G. Fytas, J. Phys. Chem. B 113, 3355 (2009) (document)

6. T. Cherdhirankorn, V. Harmandaris, A. Juhari, P. Voudouris, G. Fytas, K. Kremer, K. Koynov, Macromolecules 13, 4858 (2009) (document)

7. P. Gallo, M. Rovere, J. Phys.: Condens. Matter 15, 7625 (2003) (document)

8. P. Dieterich, R. Klages, R. Preuss, A. Schwab, Proc. Natl. Acad. Sci. 105, 259 (2008) (document)

9. I. Wong, M. Gardel, D. Reichman, E. Weeks, M. Valentine, A. Bausch, D. Weitz, Phys. Rev. Lett. 92, 178101 (2004) (document)

10. I.M. Tolić-Nørrelykke, E.L. Munteanu, G. Thon, L. Oddershede, K. Berg-Sørensen, Phys. Rev. Lett. 93, 078102 (2004) (document)

11. D.S. Banks, C. Fradin, Biophys. J. 89, 2960 (2005) (document)

12. J. Szymanski, M. Weiss, Phys. Rev. Lett. 103(3), 038102 (2009). DOI 10.1103/PhysRevLett.103.038102 (document)

13. M.J. Saxton, Biophysical Journal 70, 1250 (1996) (document)

14. G. Fytas, Personal communication (document)

15. J. Sprakel, J. van der Gucht, M.A. Cohen Stuart, N.A.M. Besseling, Phys. Rev. Lett. 99(20), 208301 (2007). DOI 10.1103/PhysRevLett.99.208301 (document)

16. T. Hu, A.Y. Grosberg, B.I. Shklovskii, Biophys. J. 90(8), 2731 (2006). DOI 10.1529/biophysj.105.078162 (document)

17. O.G. Berg, R.B. Winter, P.H. von Hippel, Biochemistry 20, 6929 (1981) (document)

18. P.J. Flory, Principles of Polymer Chemistry (Cornell University Press, Ithaca, NY, 1953) (document)

19. P. Pincus, Macromolecules 9, 386 (1976). DOI 10.1021/ ma60051a002 (document)

20. P.G. de Gennes, Scaling Concepts in Polymer Physics (Cornell University Press, Ithaca, NY, 1979) (document)

21. M.P. Allen, D.J. Tildesley, Computer Simulation of Liquids, 1st edn. Oxford Science Publications (Clarendon Press, Oxford, 1987) (document)

22. J.T. Padding, A.A. Louis, Phys. Rev. E: Stat., Nonlinear, Soft Matter Phys. 74(3), 031402 (2006). DOI 10.1103/ PhysRevE.74.031402 (document)

23. A.I. Milchev, in Computer simulations of surfaces and interfaces, vol. 114, ed. by A.I.M. Burkhard Dünweg, David P. Landau (Kluwer Academic Publishers, 2002), vol. 114 (document)

24. H.J. Limbach, A. Arnold, B.A. Mann, C. Holm, Comp. Phys. Comm. 174(9), 704 (2006). DOI 10.1016/j.cpc.2005.10.005 (document)

25. D. Ben-Avraham, S. Havlin, Diffusion and reactions in fractals and disordered systems (Cambridge University Press, 2000) (document)

26. R. Klages, in Reviews of Nonlinear Dynamics and Complex$i t y$, vol. 3, ed. by H.G. Schuster (Wiley-VCH, 2010), vol. 3 , pp. 169-216 (document) 